\title{
La expansión urbana y sus impactos sobre el clima y la sociedad de la ciudad de Bahía Blanca, Argentina
}

\section{Urban growth and its impacts on the climate and the society of Bahía Blanca city, Argentina}

\author{
Federico Ferrelli, María Luján Bustos y María Cintia Piccolo ${ }^{1}$
}

\begin{abstract}
RESUMEN
El objetivo de este trabajo fue analizar los efectos de la expansión urbana de Bahía Blanca sobre el microclima y la biomasa considerando la percepción de sus habitantes. Se utilizaron imágenes satelitales LANDSAT para el período 1986-2014. Se estimó el área urbana, la temperatura y la biomasa. Se analizaron datos meteorológicos de temperatura, humedad relativa y velocidades máximas de viento provistos por el Servicio Meteorológico Nacional (Argentina). El crecimiento de la ciudad redujo la biomasa. La TST disminuyó $0.6^{\circ} \mathrm{C}$ debido al aumento de las sombras generadas por los edificios, a la densidad de los mismos y a la capacidad calorífica de los materiales que los componen, entre otros factores. La temperatura del aire se incrementó $0.7^{\circ} \mathrm{C}$ y la humedad relativa disminuyó un $6 \%$. La opinión de los habitantes coincidió con los datos meteorológicos analizados. La consulta a los actores sociales generó información útil para orientar la planificación de la ciudad, las políticas públicas y la toma de decisiones para mejorar la calidad de vida de los habitantes.
\end{abstract}

${ }^{1}$ El primer autor forma parte del Departamento de Geografía y Turismo de la Universidad Nacional del Sur (12 de octubre y San Juan, $4^{\circ}$ piso, B8000FWB Bahía Blanca, Buenos Aires, Argentina); la segunda autora forma parte del Instituto Argentino de Oceanografía, Consejo Nacional de Investigaciones Científicas y Técnicas (IADO-CONICET), (Florida 8000. Complejo CCT CONICET, Edificio E1. B8000FWB Bahía Blanca, Buenos Aires, Argentina) y la tercera autora forma parte de ambos centros. E-mail de los autores: fferrelli@criba.edu.ar; mlbustos@criba.edu.ar; ofpiccol@criba.edu.ar. 
Palabras Clave: expansión urbana; microclima; biomasa; percepción; Bahía Blanca (Argentina).

\section{Abstract}

The aim of this study was to analyze the urban growth of Bahia Blanca city and its impacts on the microclimate considering its inhabitants perception. LANDSAT satellite images were processed for the period 1984-2014. The urban area, the temperature and the biomass was estimated. Temperature, relative humidity and wind speed from the Servicio Meteorologico Nacional (Argentina) were analyzed. The growth of the city has reduced the biomass. The LST decreased $0.6{ }^{\circ} \mathrm{C}$ due to the increasing of shadows generated by buildings and the heat capacity of their materials. The opinion of the citizen corresponded with the meteorological data analyzed. Consultations with stakeholders generated useful information to guide city planning, public policy and decision making to improve the inhabitants' quality of life.

KEY WORDS: urban growth; microclimate; perception; biomass; Bahía Blanca (Argentina).

\section{INTRODUCCIÓN}

Los cambios ambientales tales como el aumento de los gases de efecto invernadero, la deforestación, la desertificación o la pérdida de biodiversidad son producto del rápido aumento de la población. La mayor parte de los habitantes se asientan en ciudades. Por lo tanto, estos espacios y sus residentes son los factores claves para estudiar el cambio ambiental global (Grimmond, 2007).

La urbanización provoca una alteración de los procesos aerodinámicos, térmicos e hidrográficos que ocurren en la atmósfera local generando una modificación artificial del clima, denominado clima urbano (Lombardo, 1997). Existen varios factores que actúan como condicionantes de este tipo de clima: el clima regional, el tiempo atmosférico, la topografía, tamaño y morfología de la ciudad, el reemplazo de un espacio natural por otro, las diferencias en las constantes físicas de los materiales urbanos, la impermeabilización de la superficie natural, la falta o escasez de superficies evaporativas, la composición del aire urbano, el crecimiento de la ciudad, etc. (Pérez et al., 2003; Rosenzweig et al., 2005; Wong y Yu, 2005; Chen et al., 2006; Yuan y Bauer, 2007; Santana, 2007).

Por otro lado, las ciudades intervienen en el calentamiento global e impactan sobre el balance de calor debido a que son las principales emisoras de $\mathrm{CO}_{2}$ a la atmósfera (actividad industrial, tránsito, entre otros) (Velasco y Roth, 2010). El crecimiento de las mismas modifica la distribución de la Tempera- 
tura de Superficie Terrestre (TST) y resalta la importancia de incluir espacios verdes. El aumento de áreas arboladas reduce el impacto ambiental que genera el proceso de urbanización regulando la temperatura, conservando energía, agua y mejorando la calidad del aire (Wong y Yu, 2005; Capelli de Steffens et al., 2005). Por ello, la vegetación está relacionada con la TST y la temperatura del aire ya que cuando se modifica la cobertura vegetal hay una reducción de la mitigación del calor solar (Dimoudi y Nikolopoulou, 2003; Oltra-Carrió et al., 2010). El Índice Normalizado de Vegetación (NDVI) se toma como un indicador de la variación y el cambio que la TST presenta durante un período de tiempo (Goetz et al., 2000).

La expansión urbana produce cambios en la temperatura del aire que pueden afectar el confort de los residentes (Deosthali, 1999; Bustos y Piccolo, 2012). Esto genera la necesidad disponer de información social para conocer el impacto de la variabilidad climática sobre la población y su espacio. Los estudios actuales se enfocan en analizar la actitud de las personas frente a la variabilidad climática y sus estrategias de adaptación ya que son fundamentales para el desarrollo de políticas públicas. La percepción climática y el estudio social del cambio climático se ha desarrollado ampliamente en los últimos años (Owoeye y Ogunleye, 2015; Gharagozlo, 2015). De esta manera se pueden citar quienes proponen métodos descriptivos (Malone y Reyner, 2001; Pardo, 2007), quienes estudian la resiliencia social (Dietz et al., 2003), las formas de organizarse (Berkhout et al., 2006) y el capital social (Adger, 2003). Los ciudadanos son los actores sociales que intervienen sobre el espacio generando modificaciones para adaptarse a los cambios climáticos (Oltra et al., 2009).

Por lo mencionado, se considera que el estudio del clima urbano como recurso, contexto y amenaza es importante para el desarrollo de la ciudad. El mismo permite conocer las aptitudes y restricciones climáticas con vista a futuras expansiones urbanísticas. Además, es necesario comprender las problemáticas de los habitantes producto de estos cambios con el fin de orientar la toma de decisiones hacia la mejora de la calidad de vida. Por tal motivo, el objetivo de este trabajo fue analizar la evolución de la expansión urbana y sus impactos sobre la TST, la temperatura del aire, la humedad relativa, la velocidad máxima del viento, la biomasa y la percepción de la población de la ciudad de Bahía Blanca (Argentina) frente a estos cambios durante el período 1986-2014.

\section{Área de estudio}

Bahía Blanca se localiza en el sudoeste de la provincia de Buenos Aires, Argentina ( $38^{\circ} 35^{\prime} \mathrm{S}, 62^{\circ} 13^{\prime} \mathrm{W}$ ) y es capital del partido homónimo (figura 1). 
Posee una población de 301.531 habitantes (INDEC, 2010). Es el segundo centro portuario más importante de la Argentina, característica que la convierte en la ciudad principal de la región.

Está localizada sobre el margen derecho del estuario que lleva su nombre. Se enmarca dentro de los climas templados con veranos e inviernos intensos y primaveras y otoños moderados. La temperatura media anual oscila entre $14 \mathrm{y}$ $20^{\circ} \mathrm{C}$ y las precipitaciones de la región decrecen en sentido noreste - sudoeste desde $870 \mathrm{~mm}$ hasta $380 \mathrm{~mm}$. La precipitación media anual de la ciudad es de $650 \mathrm{~mm}$ (Campo de Ferreras et al., 2004). Los valores altimétricos oscilan entre los 8 y los $74 \mathrm{msnm}$ en sentido norte-sur. Los barrios al norte se localizan en un nivel de terraza que supera los $60 \mathrm{~m}$. El arbolado urbano es discontinuo y en ciertas arterias es inexistente. La ciudad posee dos parques principales con vegetación arbórea, el Parque de Mayo y el Independencia, ambos son atravesados por el arroyo Napostá que en ciertos sectores ha sido entubado (Capelli et al., 2005) (figura 1).

FIGURA 1

LOCALIZACIÓN Y PLANO DE LA CIUDAD DE BAHÍA BLANCA

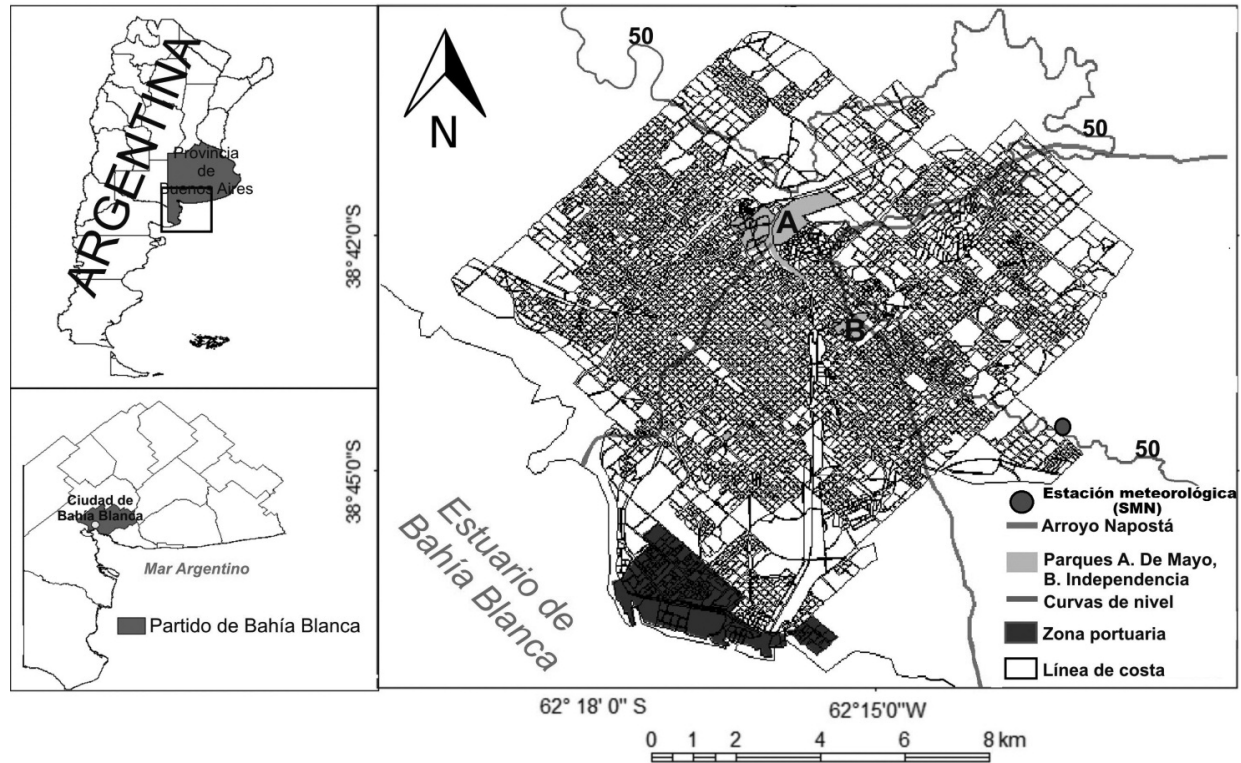

Estudios Geográficos, Vol. LXXVII, 281, pp. 469-489, julio-diciembre 2016 ISSN: 0014-1496, eISSN: 1988-8546, doi: 10.3989/estgeogr.201615 


\section{Metodología}

Para el desarrollo de este trabajo se utilizaron imágenes satelitales LANDSAT 5 TM, 7 ETM + y 8 OLI-TIRS correspondientes al período 1986-2014. Las mismas presentan una resolución espacial de $30 \mathrm{~m}$ para las bandas del visible y $120 \mathrm{~m}$ en las bandas del térmico del LANDSAT 5 TM y 7 ETM + y 100 m para el 8 OLI-TIRS. A la latitud en la que se ubica el área de estudio este satélite registró información a las 10:43 hora local, por lo que permitió el estudio de la isla de frescor diurna.

Estas imágenes fueron proporcionadas por la Comisión Nacional de Actividades Espaciales (CONAE) de Argentina y el United Stated Geological Survey (USGS) de Estados Unidos. La representación de los resultados se realizó dividiendo el período de estudio (28 años) en 4 rangos: 1986-1992, 1993-1999, 2000-2006, 2007-2014.

La expansión urbana se estimó a partir de interpretación visual. Con las imágenes LANDSAT 5 TM y 7 ETM+ se realizó una combinación de colores RGB (Red-Green-Blue) 7-4-2, mientras que con las del LANDSAT 8 OLI-TIRS, la misma fue RGB 7-6-4. Estos falsos colores permitieron observar el área urbana de color magenta, haciendo que los espacios construidos tengan contraste con su entorno. El estudio del crecimiento urbano se relacionó con el Índice Normalizado de Vegetación (NDVI). El mismo permitió analizar los cambios en la biomasa del interior de la ciudad. Para ambos cálculos se utilizaron las imágenes correspondientes a los meses de primavera del hemisferio sur (septiembre, octubre y noviembre). Según lo expresado por Lo y Quattrochi (2003) la primavera es el momento en el que la vegetación comienza a crecer y, por ende, el período más adecuado para estudiarla con imágenes LANDSAT. Las que fueron seleccionadas para este apartado se presentan en la tabla 1. Cabe señalar que las pertenecientes al LANDSAT 7 ETM + fueron corregidas debido al error de escaneo aplicando la técnica de interpolación SLCOff (Scan Line Corrector) (Pringle et al., 2009).

La TST se define como la energía de onda larga que es emitida por la superficie terrestre. Los valores de este parámetro dependen de la composición de la superficie, de la humedad relativa, la rugosidad y el tamaño de los elementos (Valor y Caselles, 1996; Rivas y Caselles, 2004). En este sentido, se consideró a este parámetro como un estimador del aumento de los edificios en altura, dado que la rugosidad que generan los mismos hace que se produzcan más sombras y con ello afecten sus valores. Teniendo en cuenta lo mencionado, se analizaron las variaciones de la distribución espacial de la TST con imágenes satelitales correspondientes a días representativos de primavera, verano, 
otoño e invierno para 1986 y 2014. Los criterios adoptados para la selección de las fechas fueron: libre de nubosidad, presión atmosférica estable, velocidad del viento inferiores a $10 \mathrm{~km} \mathrm{~h}^{-1}$, temperatura y humedad relativa similar para ambos años. Las mismas permitieron analizar la Isla de frescor superficial de la mañana y los cambios en el parámetro producto de la expansión urbana. Las imágenes seleccionadas para este estudio se presentan en la tabla 2.

TABLA 1

IMÁGENES SATELITALES UTILIZADAS PARA EL ANÁLISIS DE LA EXPANSIÓN URBANA Y EL NDVI

\begin{tabular}{llll}
\hline \multicolumn{1}{c}{ Satélite } & \multicolumn{1}{c}{ Fecha } & Fuente & Path-Row \\
\hline L5 TM & 24 de septiembre de 1986 & USGS & $226-087$ \\
L5 TM & 27 de septiembre de 1987 & USGS & $226-087$ \\
L5 TM & 16 de octubre de 1988 & USGS & $226-087$ \\
L5 TM & 18 de octubre de 1989 & USGS & $226-087$ \\
L5 TM & 21 de octubre de 1990 & USGS & $226-087$ \\
L5 TM & 8 de octubre de 1991 & CONAE & $226-087$ \\
L5 TM & 26 de octubre de 1992 & USGS & $226-087$ \\
L5 TM & 11 de septiembre de 1993 & USGS & $226-087$ \\
L5 TM & 17 de noviembre de 1994 & USGS & $226-087$ \\
L5 TM & 8 de septiembre de 1995 & USGS & $226-087$ \\
L5 TM & 19 de septiembre de 1996 & CONAE & $226-087$ \\
L5 TM & 9 de noviembre de 1997 & USGS & $226-087$ \\
L5 TM & 27 de octubre de 1998 & USGS & $226-087$ \\
L5 TM & 28 de octubre de 1999 & CONAE & $226-087$ \\
L7 ETM + & 3 de noviembre de 2000 & CONAE & $226-087$ \\
L7 ETM + & 20 de noviembre de 2001 & CONAE & $226-087$ \\
L7 ETM + & 30 de octubre de 2002 & USGS & $226-087$ \\
L7 ETM + & 2 de noviembre de 2002 & USGS & $226-087$ \\
L5 TM & 26 de noviembre de 2003 & USGS & $226-087$ \\
L5 TM & 9 de septiembre de 2004 & USGS & $226-087$ \\
L5 TM & 12 de septiembre de 2005 & USGS & $226-087$ \\
L5 TM & 15 de septiembre de 2006 & USGS & $226-087$ \\
L5 TM & 2 de septiembre de 2007 & USGS & $226-087$ \\
L5 TM & 6 de octubre de 2008 & USGS & $226-087$ \\
L5 TM & 23 de septiembre de 2009 & USGS & $226-087$ \\
L5 TM & 12 de octubre de 2010 & USGS & $226-087$ \\
L5 TM & 29 de octubre de 2011 & USGS & $226-087$ \\
L7 ETM + & 10 de noviembre de 2012 & USGS & $226-087$ \\
L8 OLI-TIRS & 20 de octubre de 2013 & USGS & $226-087$ \\
L7 ETM + & 13 de septiembre de 2014 & USGS & $226-087$ \\
\hline
\end{tabular}

Estudios Geográficos, Vol. LXXVII, 281, pp. 469-489, julio-diciembre 2016

ISSN: 0014-1496, eISSN: 1988-8546, doi: 10.3989/estgeogr.201615 
TABLA 2

IMÁGENES SATELITALES SELECCIONADAS PARA EL ESTUDIO DE LA VARIACIÓN ESTACIONAL DE LA TST EN LA CIUDAD DE BAHÍA BLANCA DURANTE 1986 Y 2014

\begin{tabular}{llc}
\hline & $1986($ L5 TM) & $2014($ L8 OLI - TIRS $)$ \\
\hline Verano & 27 de enero & 8 de enero \\
Invierno & 22 de julio & 19 de julio \\
Otoño & 16 de marzo & 14 de abril \\
Primavera & 24 de septiembre & 13 de septiembre (L7 ETM+) \\
\hline
\end{tabular}

La TST se estimó mediante el método Monocanal. El mismo se basa en la Ecuación de Transferencia Radiativa (ETR) (Jimenez-Muñoz et al., 2009):

$$
\mathrm{L}_{\text {sen }}=\left[\varepsilon B\left(\mathrm{~T}_{\mathrm{S}}+(1-\varepsilon) \mathrm{L}_{\mathrm{d}}\right] \tau+\mathrm{L}_{\mathrm{u}}\right.
$$

donde:

$\mathrm{L}_{\mathrm{sen}}$ : Radiancia medida por el sensor $\left(\mathrm{W} \mathrm{m} \mathrm{m}^{-2} \mathrm{sr}^{-1} \mu \mathrm{m}^{-1}\right)$

$\varepsilon$ : Emisividad de la superficie.

B: Parámetro derivado de la Ley de Planck calculado a partir de la ecuación [3]

$\mathrm{T}_{\mathrm{S}}$ : Temperatura de la superficie terrestre $\left({ }^{\circ} \mathrm{K}\right)$

$\mathrm{L}_{\mathrm{d}}$ : Radiancia atmosférica decendente $\left(\mathrm{W} \mathrm{m} \mathrm{m}^{-2} \mathrm{sr}^{-1} \mu \mathrm{m}^{-1}\right)$

$\tau$ : Transmisividad atmosférica

$\mathrm{L}_{\mathrm{u}}$ : Radiancia atmosférica ascendente $\left(\mathrm{W} \mathrm{m} \mathrm{m}^{-2} \mathrm{sr}^{-1} \mu \mathrm{m}^{-1}\right)$

Para obtener $L_{u}, L_{d} y \tau$, necesarios para completar la fórmula [2], se utilizaron los datos de radiosondeo de la National Aeronautics and Space Administration (NASA) vía online (http://atmcorr.gsfc.nasa.gov). Para su estimación, se obtuvieron los datos de hora de la imagen, fecha, presión, altura, temperatura del aire y humedad relativa.

Los valores de TST se calcularon con la inversión directa de la ETR (Jimenez-Muñoz et al., 2009):

$$
B\left(T_{S}\right)=\frac{\left(\mathrm{L}_{\text {sen }}-\mathrm{L}_{\mathrm{u}}-\tau(1-\varepsilon) \mathrm{L}_{\mathrm{d}}\right)}{\tau \varepsilon}
$$

Finalmente para resolver la ecuación [2], se calibró radiométricamente la banda del espectro térmico. Sobre estos valores se calculó la emisividad de la 
superficie considerando una superficie heterogénea tanto en temperatura como en emisividad y no se consideró la influencia de la sombra. El error obtenido fue $0.1{ }^{\circ} \mathrm{C}$ y se asumió un sistema lambertiano (Valor y Caselles, 1996).

$$
\varepsilon=\varepsilon_{\mathrm{v}} \mathrm{P}_{\mathrm{v}}+\varepsilon_{\mathrm{s}}\left(1-\mathrm{P}_{\mathrm{v}}\right)
$$

donde $\varepsilon_{\mathrm{v}}$ y $\varepsilon_{\mathrm{p}}$ corresponden a los valores de emisividad de la vegetación y el suelo respectivamente. $\mathrm{P}_{\mathrm{v}}$ es la proporción de vegetación y se calcula en base a la función del NDVI.

Se aplicaron correcciones en el espectro solar para estimar el NDVI. Para las bandas del visible e infrarrojo cercano. Se estimó la reflectividad considerando una superficie uniforme Lambertiana, con condiciones libres de nubes, mediante la siguiente ecuación (Schroeder et al., 2006):

$$
\mathrm{P}_{\lambda \mathrm{S})}=\frac{[\pi(\mathrm{L} \lambda \mathrm{sat}-\mathrm{L} \lambda \mathrm{p})]}{\mathrm{T}_{\lambda \mathrm{v}}\left(\mathrm{E}_{\lambda_{\mathrm{o}}} \mathrm{d}^{-2} \cos \theta_{\mathrm{z}} \mathrm{T}_{\lambda \mathrm{z}}+\mathrm{E}_{\text {down }}\right)}
$$

donde:

$\mathrm{L}_{\lambda p}\left(\mathrm{~W} \mathrm{~m}^{-2} \mathrm{sr}^{-1} \mu \mathrm{m}^{-1}\right)$ es la radiancia registrada como resultado de la interacción entre la radiación electromagnética y los componentes atmosféricos,

$\mathrm{T}_{\lambda_{v}}$ Trasmisividad atmosférica desde la superficie hacia el sensor,

$\mathrm{T}_{\lambda z}$ Trasmisividad atmosférica en la dirección de la iluminación solar y,

$\mathrm{E}_{\text {down }}$ Irradiancia difusa del cielo hacia abajo $\left(\mathrm{W} \mathrm{m}^{-2} \mu \mathrm{m}^{-1}\right)$.

Para calcular los parámetros $\left(\mathrm{L}_{\lambda p}, \mathrm{~T}_{\lambda v}, \mathrm{~T}_{\lambda z} \mathrm{y} \mathrm{E}_{\text {down }}\right)$ se aplicó el método de Resta de Superficies Oscuras (Song et al., 2001). El mismo supone que en la imagen se encuentran píxeles con una reflectividad cercana a cero (zonas oscuras) como lo son las aguas claras o profundas, zonas altamente forestadas o regiones de sombras. En estos píxeles es posible observar los efectos de la atmósfera (Carmona et al., 2011).

Una vez realizadas las correcciones en las bandas del visible, se calculó el NDVI a partir de la ecuación:

$$
\mathrm{NDVI}=\frac{\mathrm{IR}-\mathrm{R}}{\mathrm{IR}+\mathrm{R}}
$$

donde $R$ corresponde a la banda del Rojo e IR a la banda del Infrarrojo cercano.

El Servicio Meteorológico Nacional (SMN, Argentina) proporcionó datos meteorológicos diarios para el análisis de los parámetros de temperatura del aire y humedad relativa del período 1984-2012. Los datos de la velocidad má- 
xima del viento se analizaron para el período 1984-2009. Se utilizaron técnicas estadísticas estándar. Con los mismos se estimaron las anomalías anuales y se calculó la tendencia de los parámetros para el período de estudio.

Finalmente y con el objetivo de analizar la percepción del habitante de diferentes vecindarios de la ciudad y su relación con el análisis climático realizado, se efectuaron 177 cuestionarios analíticos. El mismo incluyó preguntas cerradas y abiertas. Se aplicaron en áreas distribuidas en toda la ciudad seleccionadas al azar utilizando la técnica de muestreo aleatorio simple. Este permitió conocer la percepción de la población sobre el estado ambiental actual de la ciudad y sobre los efectos de la variabilidad climática..Estuvieron comprendidos por 17 preguntas abiertas y cerradas. Se indagó en el por qué de las respuestas para obtener datos sobre percepción de los habitantes. En general los cuestionados fueron personas adultas, residentes transitorios o habitantes. En total fueron dirigidos a 101 mujeres y 76 hombres.

\section{RESULTADOS Y DISCUSIÓN}

\section{El crecimiento urbano y su relación con el NDVI}

En 1980 la población de Bahía Blanca fue de 234.047 habitantes y en 2010 de 301.531 (INDEC, 2010). En este período se observó un aumento del 22,4\% (64.484 habitantes). El crecimiento del área urbana de la ciudad también fue constante durante el período analizado. En 1986, la ciudad tenía una extensión de 52,8 km². En el año 2014 registró $111,1 \mathrm{~km}^{2}$ (un incremento del $110 \%$ ), por lo que el aumento medio anual fue de $2 \mathrm{~km}^{2}$. En 1993-1999, se observó una expansión del área construida con respecto a 1986-1992. La ciudad se extendió hacia el noreste y este. En 2000-2006, se identificó una mayor expansión del área urbanizada hacia el norte, noreste y sudeste. Finalmente, en 2007-2014 se edificó en sentido sudeste, norte y noreste (figura 2). Entre los períodos 1986-1992 y 1993-1999 el área urbana creció un 36,6\%, siendo el mayor incremento de todo el período. El menor se registró entre 2000-2006 y $2007-2014$ con un $23 \%$.

El NDVI permitió analizar la disponibilidad y el estado de la biomasa en el interior de la ciudad. Los valores del índice fueron menores en la zona urbana que en su periferia. Esto podría relacionarse con el aumento de las sombras, con las propiedades físicas de los materiales de las construcciones, con el volumen del área construida y con la reducción de la cobertura natural y el arbolado urbano (figura 3). 
FIGURA 2

EXPANSIÓN URBANA DE LA CIUDAD DE BAHÍA BLANCA DURANTE EL PERÍODO 1986-2014 CALCULADA A PARTIR DE INTERPRETACIÓN VISUAL DE IMÁGENES LANDSAT

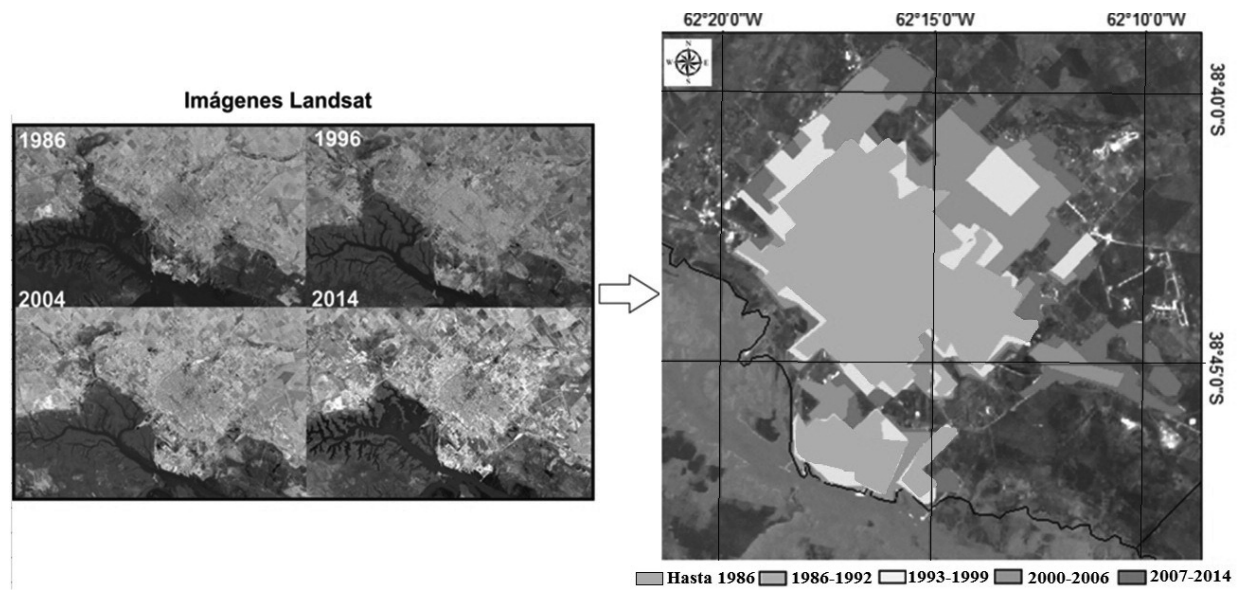

FIGURA 3

EVOLUCIÓN DE LA ZONA CONSTRUIDA Y DEL VALOR MEDIO DEL NDVI EN LA CIUDAD DE BAHÍA BLANCA PARA EL PERÍODO 1986-2014

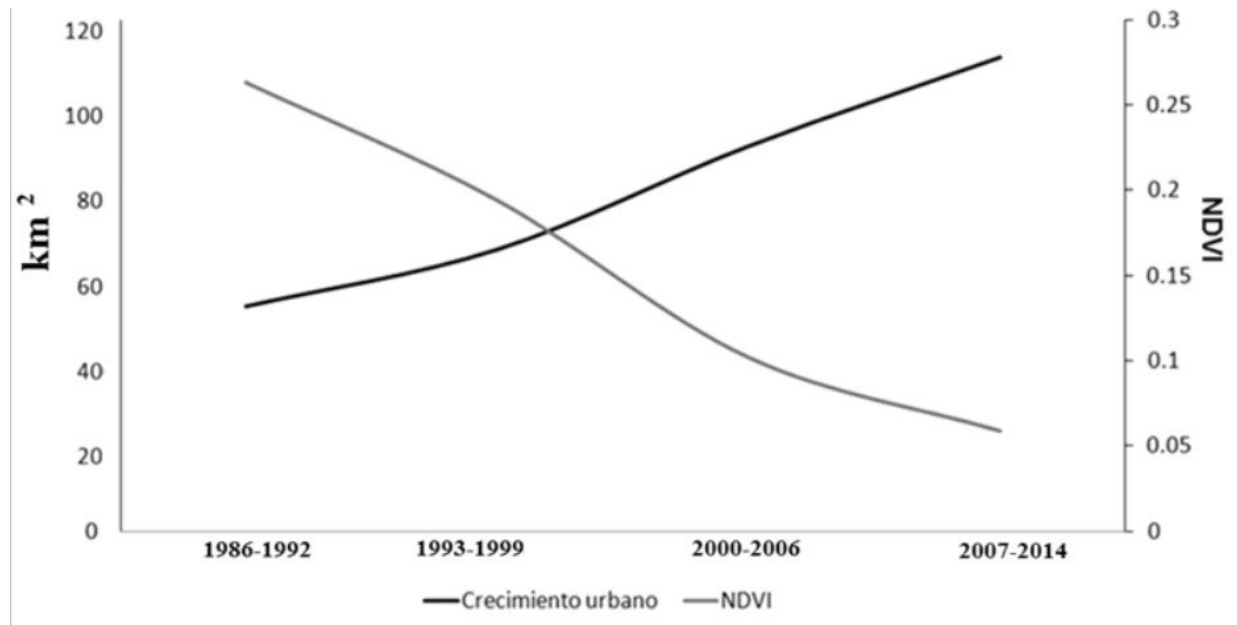

Estudios Geográficos, Vol. LXXVII, 281, pp. 469-489, julio-diciembre 2016 ISSN: 0014-1496, eISSN: 1988-8546, doi: 10.3989/estgeogr.201615 
El NDVI decreció en sentido norte y sudeste, coincidente con el aumento del espacio construido. Durante los dos primeros períodos analizados (19861992 y 1993-1999) el comportamiento del índice fue homogéneo y presentó valores positivos. A partir de 2004 y hasta 2014 este índice presentó valores negativos en el centro de la ciudad (figura 4). Tal como afirman Luber y McGeehin (2008), el cambio de las coberturas vegetales a suelos pavimentados provoca una modificación en la capacidad de almacenamiento térmico, de la que resulta una alteración que puede ser significativa para el área urbana en relación a su entorno. La ausencia de vegetación altera la distribución espacial de la humedad relativa y la energía que se desprende de los edificios durante la noche ocasionan una fuente artificial de calor que da lugar a la formación de islas de calor (Capelli de Steffens et al., 2005).

\section{FIGURA 4}

DISTRIBUCIÓN ESPACIAL DEL NDVI EN DISTINTOS AÑOS PARA LA CIUDAD DE BAHÍA BLANCA

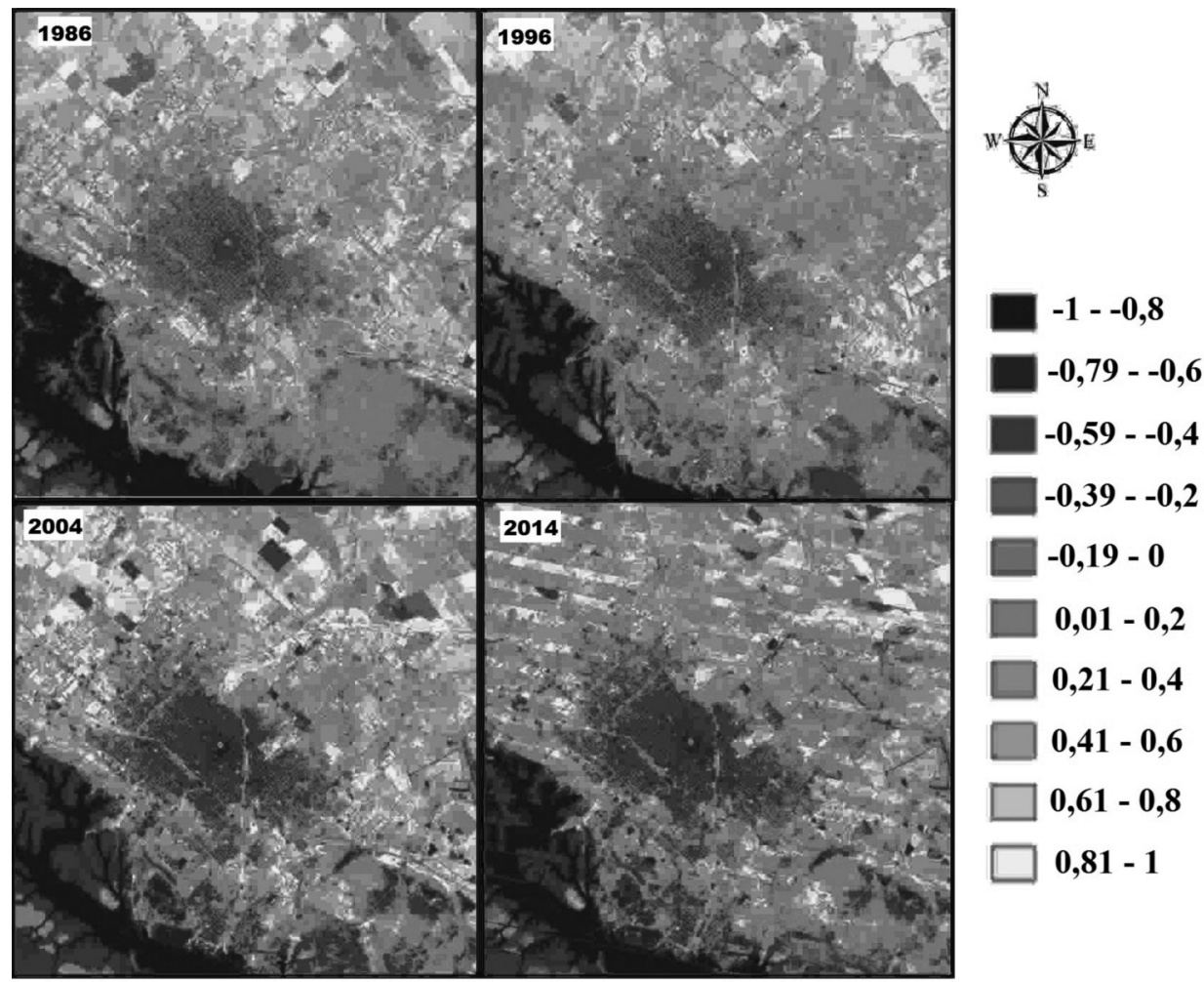

Estudios Geográficos, Vol. LXXVII, 281, pp. 469-489, julio-diciembre 2016 ISSN: 0014-1496, eISSN: 1988-8546, doi: 10.3989/estgeogr.201615 


\section{Impactos del crecimiento urbano sobre la TST}

Las mayores variaciones primaverales de la TST se observaron en los meses en los que el ángulo de elevación solar fue mayor (fines de octubre y noviembre) ya que de esta depende el tamaño de las sombras generadas. En el año 2000, por ejemplo, el día 20 de noviembre registró una amplitud térmica de $24,4^{\circ} \mathrm{C}$ y la elevación solar fue la mayor, con $54,6^{\circ}$.

El valor medio de las amplitudes térmicas fue $14,6^{\circ} \mathrm{C}$. El mismo sólo fue superado en 10 de las fechas analizadas. El mínimo valor fue $5,8^{\circ} \mathrm{C}$ en 1986 y el mayor fue $23,5^{\circ} \mathrm{C}$ en 2000 . A la hora del paso del satélite por la ciudad de Bahía Blanca se encontró que las áreas rurales fueron más cálidas que las urbanas, es decir que la periferia presentó mayores temperaturas que el centro de la ciudad. Esto se debe a que por la mañana la estructura de las calles y de los edificios provocaron que el centro de la urbe se mantenga a la sombra mientras que los alrededores de la ciudad ya están siendo calentados por el sol (Valor et al., 2000). El diferente trazado y morfología de ambos espacios tiene un rol fundamental. Es importante destacar que el comportamiento de la TST está influenciado por las condiciones micro-climáticas relacionadas con la estructura urbana, el uso del suelo, la morfología y su orientación (López Gómez et al., 1990). Las áreas sin vegetación presentaron valores altos de la TST de superficies iluminadas por el sol, como el suelo desnudo o pavimentado (Weng et al., 2004).

A pesar de que la tendencia de la TST fue siempre la misma, con un centro frío y la periferia más cálida, sus variaciones dependieron de la densidad de las edificaciones. Entre 1986 y 2014 se acentuaron las diferencias de la TST entre las áreas construidas y la periferia. Las zonas más edificadas presentaron temperaturas más bajas respecto de las zonas periféricas donde la temperatura fue más alta y homogénea. En general, entre los años mencionados, se presentó una tendencia decreciente de $0,6^{\circ} \mathrm{C}$. Lo mismo ocurrió con los valores mínimos $\left(-2^{\circ} \mathrm{C}\right)$. Sin embargo, los valores máximos se incrementaron $2,7^{\circ} \mathrm{C}$ en todo el período.

La distribución estacional de la TST entre los años 1986 y 2014 fue diferente. En el verano de 1986 el centro de la ciudad y la periferia manifestaron valores similares. En el invierno, la mayor parte de la ciudad junto con la zona costera registraron los menores valores de temperatura. Las diferencias fueron más notables entre el centro de la ciudad y la periferia en todas las estaciones del año 2014. El mayor contraste fue en el invierno y los menores en verano y otoño. En el invierno, las sombras producidas por las edificaciones generaron un centro más frío que la periferia. Esto se debió a un aumento de las edifica- 
ciones en altura que presentaron más sombras generando un centro más frío. A modo de ejemplo se presenta en la figura 5 la distribución espacial de invierno y verano para ambos años. Las diferencias estacionales de los valores de TST están determinadas por la latitud, el período de insolación diferente según la estación del año y a la diferencia estacional de la radiación incidente (López Gómez et al., 1990).

\section{FIGURA 5}

DISTRIBUCIÓN ESPACIAL DE LA TST EN INVIERNO Y VERANO PARA LOS AÑOS A) 1986 Y B) 2014

\section{VERANO}
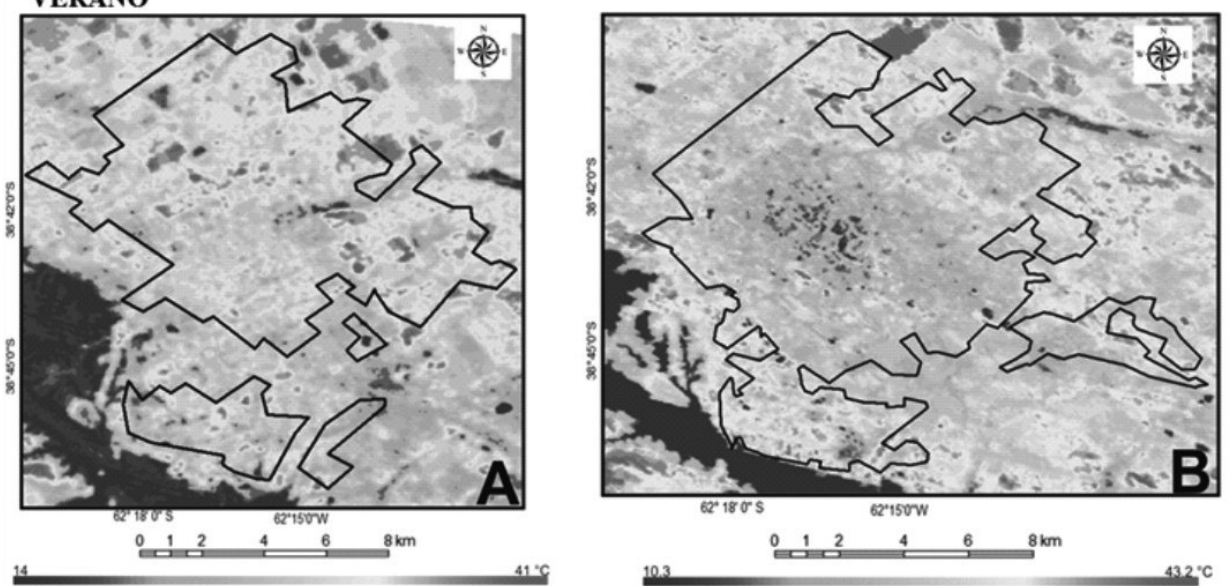

\section{INVIERNO}

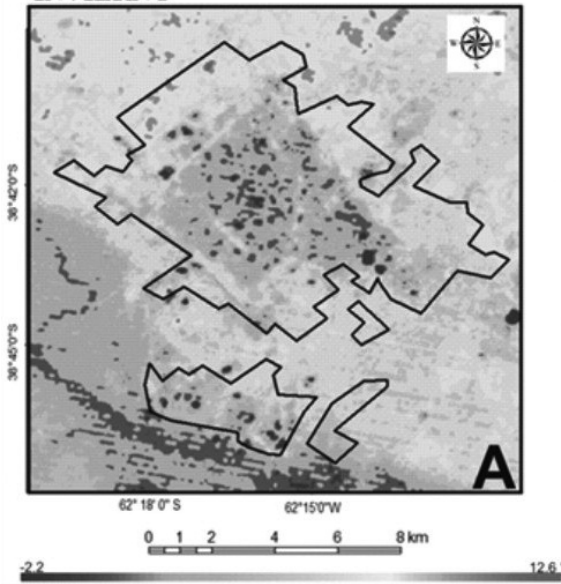

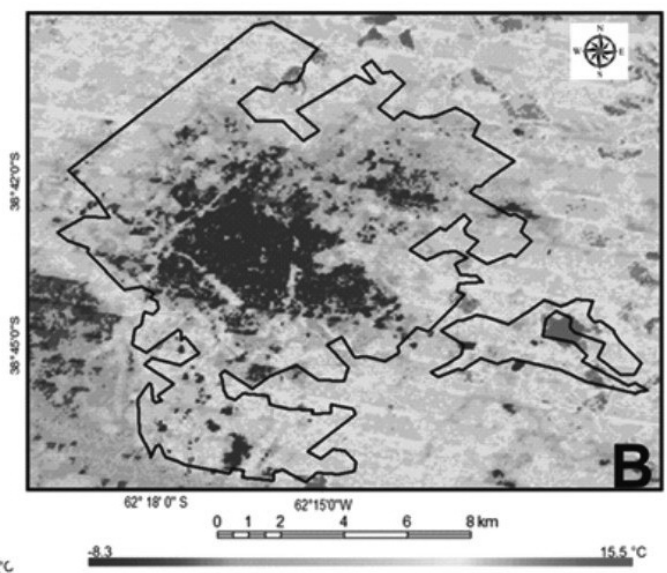


Impactos del crecimiento urbano sobre algunos parámetros meteorológicos

Las anomalías de temperatura del aire para el período 1984-2012 permitieron comprobar un aumento de $0,7^{\circ} \mathrm{C}$ entre 1984 y 2012 (figura 6) debido a la reducción de la biomasa en el interior de la ciudad. Las zonas verdes urbanas mostraron temperaturas más bajas debido a que reducen la emisión de onda larga producida por las superficies construidas sometiendo a los habitantes a reducir su carga radiante (Dimoudi y Nikolopoulou, 2003). Además, la humedad relativa fue 6\% inferior en 2012 en relación con la media anual de 1986.

Las anomalías de la velocidad máxima del viento (1984-2009) presentaron una tendencia decreciente $\left(-0,1 \mathrm{~km} \mathrm{~h}^{-1}\right.$ por año). La disminución media de la velocidad máxima del viento durante el período fue de $3,6 \mathrm{~km} \mathrm{~h}^{-1}$ (figura 7). La dirección y velocidad del viento en la ciudad de Bahía Blanca se modificó por la forma y orientación de los edificios que originan vórtices y torbellinos en ciertos sectores de la ciudad (Capelli de Steffens et al., 2005). Estos cambios en los parámetros meteorológicos estarían relacionados con el aumento del tránsito y la sustitución de suelo natural por edificaciones que modifican el albedo urbano e impactan sobre el clima de la ciudad (Sepulveda Moreno, 2006).

FIGURA 6

ANOMALÍA DE LOS VALORES MEDIOS ANUALES ANUALES DE LA TEMPERATURA DEL AIRE EN LA CIUDAD DE BAHÍA BLANCA DURANTE EL PERÍODO 1984-2012

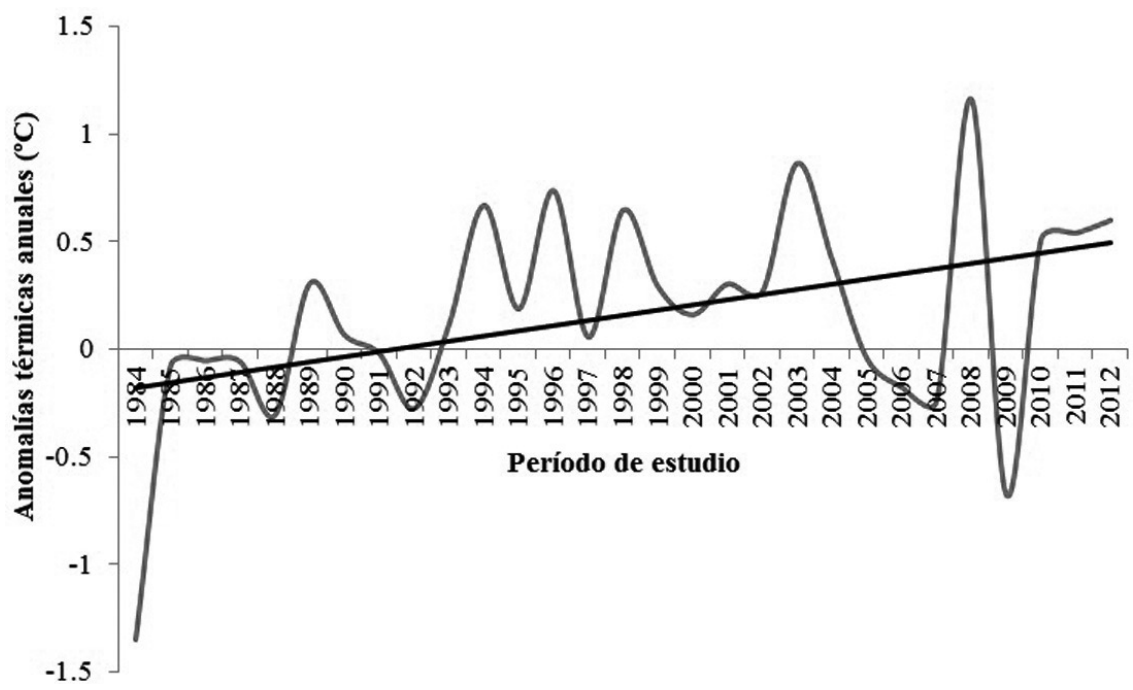

Estudios Geográficos, Vol. LXXVII, 281, pp. 469-489, julio-diciembre 2016 ISSN: 0014-1496, eISSN: 1988-8546, doi: 10.3989/estgeogr.201615 


\section{FIGURA 7}

ANOMALÍAS DE LOS VALORES MEDIOS ANUALES DE LA VELOCIDAD MÁXIMA MEDIA DEL VIENTO EN LA CIUDAD DE BAHÍA BLANCA DURANTE

EL PERÍODO 1984-2009

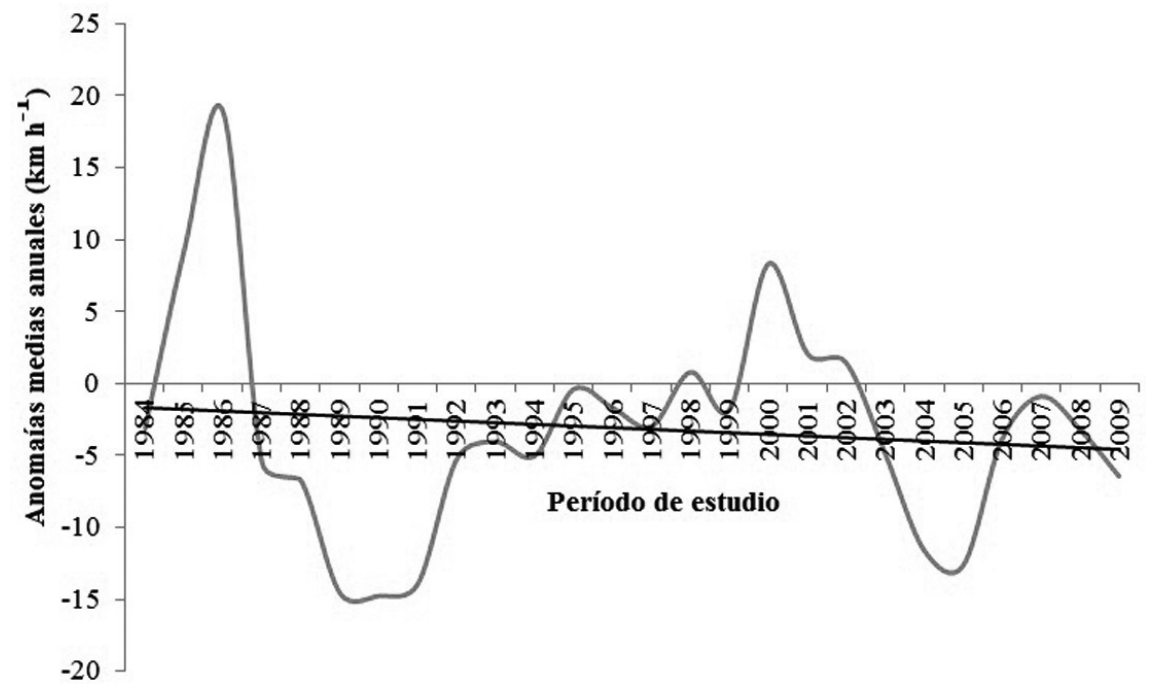

\section{Percepción de la población}

Los cuestionarios permitieron obtener información que permitió analizar la importancia que los pobladores tienen sobre los espacios verdes, los cambios en la temperatura, los eventos extremos que más incidencia tienen sobre la ciudad, el consumo energético y su relación con la variabilidad climática. Los habitantes consideraron que el estado ambiental de la ciudad es mayoritariamente Regular (58\%), en menor medida Malo y Muy Malo (31\%) y una minoría lo pensó como Bueno (11\%). En general, consideraron que la degradación ambiental se relaciona con las actividades industriales (63\%) y humanas como el aumento del tránsito vehicular (15\%), basurales a cielo abierto (8\%), actividades portuarias (3\%), expansión urbana (3\%), etc. (tabla 3).

En lo referente a los espacios verdes y a la biomasa de la ciudad, los ciudadanos (98\%) consideraron que los espacios verdes son de gran importancia para la ciudad, mientras que solo unos pocos (2\%) los consideraron como áreas de rechazo. Las principales razones positivas que le atribuyeron 
a los espacios verdes fueron que purifican el aire (43\%) y que favorecen a las actividades recreativas (31\%). También, en algunos casos, mencionaron que permiten la infiltración durante lluvias, generan sombras, mejoran la confortabilidad, regulan la temperatura del aire y mejoran la calidad ambiental (tabla 3).

El 72\% consideró que el tránsito vehicular aumentó en la ciudad de Bahía Blanca, ocasionando con ello un impacto negativo sobre el ambiente, remarcando el aumento de la contaminación sonora y del aire. Se registró también que el $75 \%$ de los encuestados percibió un aumento en el consumo energético como consecuencia de los cambios climáticos, principalmente por el aumento del uso de acondicionadores de aire y de calefacción durante los veranos e inviernos, respectivamente (tabla 3 ).

Por otro lado, la mayoría de las personas encuestadas (78\%) percibieron cambios en la temperatura del aire en los últimos años como consecuencia de la expansión urbana. Estos cambios los relacionaron a un «aumento importante» de la temperatura del aire (62\%), seguido por quienes los consideraron como un «aumento moderado». Se encontró que el $85 \%$ consideró que los eventos extremos se incrementaron en la ciudad. Los mismos están constituidos por un aumento en las precipitaciones, seguido de un mayor número de olas de calor en los veranos y olas de frío en los inviernos, en menor medida por un incremento del viento, de los períodos de sequía e inundaciones y finalmente percibieron un aumento de las tormentas eléctricas de granizo (tabla 3).

En cuanto a las medidas de adaptación o acciones a llevar a cabo para mitigar los efectos de la variabilidad climática, los encuestados propusieron un aumento de los espacios verdes $(90,2 \%)$ para reducir los efectos de la contaminación del aire, sonora y mejorar la confortabilidad climática. Además, consideraron que la calidad ambiental aumentaría con un mejor manejo de los desagües (con el fin de evitar el anegamiento de las calles) y con un aumento del arbolado urbano (para mitigar las temperaturas extremas). Por otro lado, consideraron que el arbolado urbano y de las plazas y parques de la ciudad se encuentra, en general, en mal estado por lo que requieren de mayor mantenimiento e inversión. 
TABLA 3

VARIABLES CONSIDERADAS PARA EL ANÁLISIS DE LA PERCEPCIÓN

CLIMÁTICA DE BAHÍA BLANCA

VARIABLES

\begin{tabular}{|c|c|c|c|}
\hline \multicolumn{4}{|l|}{ 1. Estado ambiental } \\
\hline Regular & \multicolumn{3}{|l|}{$58 \%$} \\
\hline Malo & \multicolumn{3}{|l|}{$20 \%$} \\
\hline Muy Malo & \multicolumn{3}{|l|}{$11 \%$} \\
\hline Bueno & \multicolumn{3}{|l|}{$11 \%$} \\
\hline \multicolumn{4}{|l|}{ 2. Causas de la degradación ambiental } \\
\hline Actividades industriales & \multicolumn{3}{|l|}{$63 \%$} \\
\hline Aumento del tránsito & \multicolumn{3}{|l|}{$15 \%$} \\
\hline Basurales a cielo abierto & \multicolumn{3}{|l|}{$8 \%$} \\
\hline Actividades portuarias & \multicolumn{3}{|l|}{$3 \%$} \\
\hline Expansión urbana & \multicolumn{3}{|l|}{$3 \%$} \\
\hline Contaminación Sonora & \multicolumn{3}{|l|}{$2 \%$} \\
\hline Poda de árboles & \multicolumn{3}{|l|}{$1 \%$} \\
\hline \multicolumn{4}{|l|}{ 3. Aumento del tránsito vehicular } \\
\hline $\mathrm{Si}$ & \multicolumn{3}{|l|}{$72 \%$} \\
\hline No & \multicolumn{3}{|l|}{$28 \%$} \\
\hline \multicolumn{4}{|c|}{ 4. Aumento del consumo energético por cambios climáticos } \\
\hline $\mathrm{Si}$ & \multicolumn{3}{|l|}{$75 \%$} \\
\hline No & \multicolumn{3}{|l|}{$25 \%$} \\
\hline \multicolumn{4}{|l|}{ 5. Cambios en la temperatura urbana } \\
\hline \multirow[t]{4}{*}{$\mathrm{Si}$} & \multirow[t]{4}{*}{$78 \%$} & Aumento significativo & $62 \%$ \\
\hline & & Aumento moderado & $33 \%$ \\
\hline & & Aumento leve & $4 \%$ \\
\hline & & disminución & $1 \%$ \\
\hline No & $22 \%$ & & \\
\hline \multicolumn{4}{|l|}{ 6. Aumento de los eventos extremos } \\
\hline Precipitaciones & \multicolumn{3}{|l|}{$38 \%$} \\
\hline Olas de calor & \multicolumn{3}{|l|}{$35 \%$} \\
\hline Aumento del viento & \multicolumn{3}{|l|}{$13 \%$} \\
\hline Olas de frío & \multicolumn{3}{|l|}{$10 \%$} \\
\hline Sequías e inundaciones & \multicolumn{3}{|l|}{$3 \%$} \\
\hline $\begin{array}{l}\text { tormentas eléctricas } \\
\text { y granizos }\end{array}$ & \multicolumn{3}{|l|}{$1 \%$} \\
\hline
\end{tabular}




\section{CONCLUSIONES}

El crecimiento urbano de la ciudad de Bahía Blanca fue de 110\% durante los 26 años analizados. Esto impactó sobre los parámetros de TST, NDVI, temperatura del aire, humedad relativa y velocidades máximas del viento. Asociado al aumento de las sombras generadas por los edificios se produjo una reducción de los valores medios de TST. A su vez, los valores de NDVI también disminuyeron. La temperatura media del aire se incrementó a lo largo del período de estudio y la velocidad máxima del viento disminuyó como consecuencia del aumento de la edificación.

La percepción de la población mantuvo una relación directa con el análisis climático y de biomasa realizado. Los encuestados consideraron que los espacios verdes son escasos y reclaman un aumento de los mismos con el fin de mejorar la calidad ambiental. El aumento de la temperatura fue percibido por el 99\% de los encuestados en diferentes intensidades. A pesar de que en Bahía Blanca se registró una disminución de la velocidad de las ráfagas, el 13\% de la población percibió un aumento en este parámetro.

Por todo lo mencionado, se considera que en la ciudad de Bahía Blanca se debe orientar la toma de decisiones y planificación de las actividades hacia la mejora y aumento de los espacios verdes. Este accionar mejorará no sólo la calidad ambiental, sino también la confortabilidad y calidad de vida de los residentes y turistas.

\section{Agradecimientos}

Este trabajo fue subvencionado por el Inter-American Institute for Global Change Research (IAI) CRN3038, el cual es apoyado por la US National Science Foundation (Grant GEO-1128040) y por el proyecto "Estudio integrado de estuarios argentinos" (24/H103) financiado por la Universidad Nacional del Sur. También se desea mencionar el apoyo del Consejo Nacional de Investigaciones Científicas y Técnicas (CONICET, Argentina). Por otro lado, se agradece al Servicio Meteorológico Nacional (SMN, Argentina), a la Comisión Nacional de Actividades Espaciales (CONAE, Argentina) y al United States Geological Survey (USGS, Estados Unidos) por brindar los datos necesarios para realizar el presente trabajo. Finalmente, a la Dra. María Andrea Huamantinco Cisneros por los aportes realizados para desarrollar esta investigación. 


\section{REFERENCIAS}

Adger, W. N. (2003): "Social Capital, Collective Action, and Adaptation to Climate Change", Economic Geography, 79, 4, pp. 387-404.

Berkhout, F., Hertin, J. y Gann, D. M. (2006): "Learning to adapt: Organizational adaptation to climate change impacts", Climatic Change, 78, pp. 135-156.

Bustos, M L. y Piccolo, M. C. (2012): "Esquema de información climática para turismo aplicado al balneario Pehuén Co, Argentina", Territoris, 8, pp. 29-40.

Campo de Ferreras, A. M., Capelli de Steffens, A. M. y Diez, G. P. (2004): El Clima del Suroeste Bonaerense, Bahía Blanca, Argentina, editorial EdiUns, 99 pp.

Capelli de Steffens, A., Piccolo, M. C. y Campo de Ferrera, A. (2005): Clima urbano de Bahía Blanca, Bahía Blanca, Argentina, Editorial Dunken, 199 pp.

Carmona, F., Rivas, R., Thomas, L. y Marino, B. (2011): "Caracterización espectral del estuario del río Quequén Grande por medio de imágenes Landsat”, en R. Rivas, F. Carmona y D. Ocampo (eds.), Teledetección. Recientes aplicaciones en la región pampeana, Mar del Plata, Argentina, Editorial Martín, pp. 11-29.

Chen, X., Zhao, H., Li, P. y Yin, Z. (2006): "Remote sensing image-based analysis of the relationship between urban heat island and land use/cover changes", Remote Sensing of Environment, 104, pp. 133-146.

Comisión Nacional de Actividades Espaciales (CONAE), http://www.conae.gov.ar (Fecha de consulta: noviembre de 2014).

Deosthali, V. (1999): "Assessment of impact of urbanization on climate: an application of bio-climatic index", Atmos Environ, 33, pp. 4.125-4.133.

Dietz, T., Ostrom, E. y Stern, P. (2003): "The Struggle to Govern the Commons", Science, 302/12, pp. 1.907-1.912.

Dimoudi, A. y Nikopoulou, M. (2003): "Vegetation in the urban environment: microclimatic analysis and benefits", Energy and Biuldings, 35, pp. 69-76.

Gharagozlo, A. R. (2015): "A survey of Tehran metropolis strategies as a creative city of Iran”, Journal of Geography and Regional Planning, 6, pp. 149-158.

Goetz, S. J., Prince, S. D. y Small, J. (2000): "Advances in Satellite Remote Sensing of Environmental Variables for Epidemiological Applications”, Adv. Parasitol., 47, pp. 289-307.

Grimmond, S. (2007): "Urbanization and global environmental change: local effects of urban warming", Geographical Journal, 173, pp. 83-88.

Instituto Nacional de Estadística y Censos (INDEC) (2011): http://www.censo2010. indec.gov.ar/ (Fecha de consulta: 14/04/2014).

Jimenez-Muñoz, J. C., Jordi, C., Sobrino, J. A., Soria, G., Ninyerola, M. y Pons, X. (2009): "Revision of the single-channel algorithm for lands surface temperature retrieval from Landsat thermal-infrared data", IEEE Transactions on geoscience and remote sensing, 47, pp. 339-349. 
Lo, C. P. y Quattrochi, D. A. (2003): "Land-use and Land-cover change, urban heat island phenomenon, and health implication: a remote sensing approach", Photogrammetric Engineering and remote Sensing, 69, pp. 1.053-1.063.

Lombardo, M. A. (1997): "Estudios interdisciplinares, metodologías e técnicas de análise do clima urbano", en VI Encuentro de Geógrafos de América Latina, Buenos Aires, Argentina, p. 298.

López Gómez A., Moreno Jiménez, A., Fernández García, F. y Palou, F. (1990): "La temperatura diurna en la Aglomeración de Madrid, mediante imágenes remotas", Revista de Estudios Geográficos, 201, pp. 705-732.

Luber, G. y McGeehing, M. (2008): "Climate change and Extreme heat events", American Journal of Preventive Medicine, 35, pp. 429-435.

Malone, E. y Rayner, S. (2001): "Role of the research standpoint in integrating globalscale and local-scale research", Climate Research, 19, pp. 173-178.

NASA, (2014): Atmospheric Correction Parameter Calculator, http://atmcorr.gsfc. nasa.gov/ (Fecha de consulta: 14/04/2014).

Oltra, C., Solà, R., Sala, R., Prades, A. y Gamero N. (2009): "Cambio climático: percepciones y discursos públicos", Revista de Ciencias Sociales, 2, pp. 1-23.

Oltra-Carrio, R., Sobrino, J. A., Gutiérrez-Angonese, J., Gioia, A, Paolini, L. y Malizia, A. (2010): "Estudio del crecimiento urbano, de la estructura de la vegetación y de la temperatura de la superficie del Gran San Miguel de Tucumán, Argentina", Revista de Teledetección, 34, pp. 69-76.

Owoeye, J. O. y Ogunleye, O. S. (2015): "Urban development and land use changes around the Ekiti State University (EKSU), Ado-Ekiti Nigeria", Journal of Geography and Regional Planning, 8, pp. 111-119.

Pardo, M. (2007): "El impacto social del cambio climático", Panorama Social, 5, pp. 22-35.

Pérez González, M., García Rodríguez, P. y Guerra Zaballos, A. (2003): "Análisis del clima urbano a partir de imágenes de satélite en el centro peninsular español", Anales de Geografía de la Universidad Complutense, 23, pp. 187-206.

Pringle, M. J., Schmidt M. y Muir, J. S. (2009): "Geostatistical interpolation of SLC-off Landsat ETM+ images", ISPRS Journal of Photogrammetry and Remote Sensing, 64, pp. 654-664.

Quattrochi, D. A. y Ridd, M. K. (1998): "Analysis of vegetation within a semi-arid urban environment using high spatial resolution airborne thermal infrared remote sensing data", Atmospheric Environment, 32, pp. 19-33.

Rosenzweig, C., Solecki, W. D., Parshall, L., Chopping, M., Pope, G. y Goldberg, R. (2005): "Characterizing the urban heat island in current and future climates in New Jersey", Environmental Hazards, 6, pp. 51-62.

Rivas, R. y Caselles, V. A. (2004): "Simplified equation to estimate spatial reference evaporation from remote sensing-based surface temperature and local meteorological data", Remote Sensing of Environment, 93, pp. 68-76.

Santana Rodriguez, L. M., Escobar Jaramillo, L. A. y Capote, P. A. (2010): "Estimación 
de un índice de calidad ambiental urbano, a partir de imágenes de satélite", Revista de Geografía Norte Grande, 45, pp. 77-95.

Sarricolea, P. y Romero Aravena, H. (2006): "Cambios de uso y coberturas en el suelo entre 1998 Y 2004 y sus efectos sobre la configuración de la isla de calor de urbana de superficie de Santiago", Repositorio Académico de la Universidad de Chile, pp. 1-4.

Schroeder, T. A., Cohen, W. B., Song, C., Canty, M. J. y Yang, Z. (2006): "Radiometric correction of multitemporal Landsat data for characterization of early successional forest patterns in western Oregon", Remote Sensing of Environment, 103, pp. 16-26.

Sepulveda Moreno, O. (2006): "Problemas ambientales de Santiago: Isla de calor y edificios de vidrios", Boletín de Geografía, 24, pp. 45-55.

Servicio Meteorológico Nacional (SMN, Argentina), http://www.smn.gov.ar/

Song, C., Woodcock, C., Seto, K. C., Pax-Lenney, M. y Macomber S. A. (2001): "Classification and change detection using Landsat TM data: when and how to correct atmospheric effects?", Remote Sensing Of Environment, 75, pp. 230-244.

United State Geological Survey (2015): http://glovis.usgs.gov/ (Fecha de consulta: 14/10/2015).

Valor, E. y Caselles, V. (1996): "Mapping land surface emissivity from NDVI: Application to European, Africa and South American areas", Remote Sensing of Environment, 57, pp. 167-184.

Valor, E., Caselles, V., Coll, C., Sánchez, F, Rubio, E. y Sospedra, F. (2000): "Análisis comparativo del efecto de isla térmica de la ciudad de Valencia con imágenes TM, MUST y AVHRR”, Revista de Teledetección, 14, pp. 1-6.

Velasco, E. y Roth, M. (2010): "Cities as net sourcer of $\mathrm{CO}_{2}$ : Review of atmospheric $\mathrm{CO}_{2}$ exchange in urban environments measured by Eddy Covariance Technique", Geography Compass, 4/9, pp. 1.238-1.259.

Weng, Q., Lu, D. y Shubring, J. (2004): "Estimation of land surface temperature - vegetation abundance relationship for urban heat island studies", Remote Sensing of Environment, 89, pp. 467-483.

Wong, N. y Yu, C. (2005): "Study of green areas and urban heat island in a tropical city", Habitat International, 29, pp. 547-558.

Yuan, F. y Bauer, M. (2007): "Comparison of impervious surface area and normalized difference vegetation index as indicators of surface urban heat island effects in Landsat imagery", Remote Sensing of Environment, 106, pp. 375-386.

Fecha de recepción: 15 de junio de 2015.

Fecha de aceptación: 11 de noviembre de 2015. 